\title{
The use of environmental sensitivity indices (ESI) maps for the evaluation of oil spill risk in Mediterranean coastlines and coastal waters
}

\author{
F. Gugliermetti ${ }^{1}$, F. Cinquepalmi ${ }^{2} \&$ D. Astiaso Garcia ${ }^{1}$ \\ 'Department of "Fisica Tecnica", Rome University "La Sapienza", Italy \\ ${ }^{2}$ Member of the National Technical Board of Marine Protected Areas of \\ the Italian Ministry for the Environment, Land and Sea Protection, Italy
}

\begin{abstract}
This paper presents a project devoted to identifying the environmental, historical and morphologically most sensitive and vulnerable priorities of the coastlines, with a view to providing decision-makers with sensitivity index maps for the planning of preventive actions and emergency responses in the event of an unforeseen outflow of hydrocarbons. The sensitivity index maps give information established by environmental sensitivity indices (ESI), represented in the maps with different lines and colours. Due to the considerable diversity in ecosystems and morphology in the Mediterranean and on account of the unique importance of the archaeological, historic and landscape sites along the Italian coastline, the decision was taken to extrapolate the ESI values on the basis of the matrices elaborated by Cinquepalmi et al (Prevention of hydrocarbons sea pollution: Sensitivity Index Maps for the Venice Lagoon as integral component of oil-spill contingency planning and response. Environmental Coastal Regions. WIT Transaction of Ecology and the Environmental volume 25. WIT Press, 1998) in a previous study with a similar aim on the Lagoon of Venice. With respect to the previous study a more simple core set of ESI was obtained from new matrices created in order to be used in the Mediterranean ecosystems: natural coastline, water stretches and artificial features. The resultant indices identify a single number that expresses all the qualitative and quantitative traits of the Italian coastline, both for land and seascapes, in clear maps of simple use. Environmental Sensitivity Maps offer a useful contribution to the prevention of pollution from oil spills and for combating the consequences of a possible accident along Mediterranean coastlines. They also constitute the most complete database, useful both for risk response and for an Integrated Coastal Zone Management.
\end{abstract}

Keywords: Integrated Coastal Zone Management, hydrocarbons pollution and oil spills, environmental sensitivity index (ESI) maps, risk assessment. 


\section{Introduction}

The major presence of hydrocarbons in the sea is connected with human activities and is a serious source of marine pollution. Oil spills can cause substantial damage to marine ecosystems, causing biodiversity loss and consequent disruption of the fragile environmental equilibrium.

Environmental sensitivity index maps are one of the possible tools for obtaining an overall picture of the vulnerability of the coastlines to accidental oil spills. These maps can in fact provide a useful contribution towards identifying major and sensitive environmental, historical and morphological priorities, providing decision-makers with a true overview with which to plan preventive action and emergency responses in the event of an accident.

One of the aims is to ensure a practical management of oil spill risk through a series of preventive actions based on forward planning both on land and sea, with a view to swiftly containing unforeseen hydrocarbon spills, thereby limiting damage to coastal environmental, cultural and economic worth. The database of information compiled through this work, however, is also extremely useful for risk response as well as for the application of the principles of Integrated Coastal Zone Management by local communities and public administrations.

\subsection{The evolution of oil spill response and risk management}

The relevance of possible contamination effects from oil are in relation to the different environmental and territorial features where they take place. Their main negative consequences are on the coastal landscape, considerably reducing its aesthetic value; on the ecosystem, provoking a drastic drop in biodiversity and consequently upsetting the environmental balance; and on activities related to man (socio-economic, historic and cultural worth).

It would therefore appear useful to draw up environmental sensitivity maps with a view to obtaining an overall picture of vulnerability factors exposed to an accidental oil spill in sea waters.

The final objective will therefore be to produce a coastal sensitivity mapping model that could be applicable to the entire Italian coastline, with a special concern for those coastal and marine areas including protected areas, considered as the test areas of the project.

The US Government has already drawn up sensitivity maps covering all the Country's sea and Great Lake coastlines, thanks to work carried out by the NOAA (National Oceanographic and Atmospheric Administration) [1], which has drawn up ESI maps for oil and chemical risk factors. The NOAA has developed a method for creating environmental sensitivity indices based only on a visual evaluation of the physical and morphological characteristics of coasts, classifying the various coastlines in whole numbers from 1 to 10 . Higher numbers are given to more vulnerable stretches of coast, identified by different colours. These environmental index maps also include information concerning flora and fauna and those resources available for local stakeholders that would be exposed to greatest risk in the event of an oil spill [2]. 
Given the considerable diversity in terms of ecosystems and morphologies along the Mediterranean coasts, not to mention the thousands of years of civilization that has sprung up and developed in the area, it has been decided to extrapolate the ESI values from the environmental sensitivity matrices, following the general methodology elaborated in the previous similar work for the Lagoon of Venice [3], within the "Venice Lagoon System Project" coordinated by Prof. A. Zitelli of the University of Architecture IUAV in Venice in 1998 [4]. Given the lack of important archaeological or historical sites along the United States coastline, the US ESI maps were less difficult to draw up since the evaluations included only ecological value and coastal-related human activities. Within the Mediterranean context the historical elements are of such inestimable value that they must be considered in a balanced evaluation process alongside the ecological aspect. The calculated/balanced values therefore have the advantage of including all the reported qualitative and quantitative coastal characteristics in a single number.

\section{Methods}

The project was implemented by the Department of Fisica Tecnica of La Sapienza University of Rome, in response to a request by General Direction of Natures Protection of The Italian Ministry for the Environment, Land and Sea Protection.

The activity carried out was oriented towards elaborating matrices suitable for the evaluation of coastal sensitivity with regards to oil spills in seawaters in different coastal contexts. Three matrices in particular were pinpointed: one for calculating the environmental sensitivity indices according to coastline morphological and natural characteristics (ESI for natural coastlines), one for calculating the ESI of water environments (ESI for water stretches), and the final matrix for evaluating sensitivity indices according to artificial elements, both above and below sea level (ESI for artificial features).

The three matrices include an overall examination of the natural or artificial elements that would be damaged by contact with hydrocarbons, as well as the overall combination of factors that alter damage risk. The ESI of each natural or artificial element is therefore extrapolated considering risk exposure factors, impact values and damage indices.

The evaluation of risk exposure factors can take into account the hydrodynamic factor (wave movement), morphology (coastal sloping and water depth, position of the buildings respect the sea level), type and incidence of surfaces that could enter in contact with polluting elements. A risk factor, corresponding to the sum of the standardised weights calculated for each factor, was considered for each category included in the matrices.

The same standardisation was carried out for the impact values, calculated according to the clean up difficulty with respect to the type of surface.

Damage indices are the integrated expression of the importance of the analysed sites, considering the presence of protected species, human activities related to economic, historical, cultural, recreational values, water quality etc. 
Damage categories have been subdivided in qualitative terms, in an integrated evaluation of all natural or artificial aspects that would be included in the negative consequences of an accident. A standardised variable value, directly related to its classification, was assigned to each characteristic examined, so as to enable a quantification of damage in direct proportion with the degree of irreversible value loss.

Environmental sensitivity index values are obtained from combining physical sensitivity indices (obtained from summing risk and impact factors) with damage risk, with each of the three matrices grouped under the different ESI classifications, from 1 to 4 . This results in an environmental sensitivity index for each of the natural or artificial elements analysed.

\subsection{ESI for natural coastlines}

The first matrix takes into account the natural and morphological aspects of coastline such as cliffs, rocky shores, beaches and mudflats.

Two factors have been identified in the evaluation of risk exposure factors, subdivided into the following classifications:

- Angle of slope. Coastlines have been subdivided into four slope levels; each slope class correspond to a risk classification that is inversely proportioned to the slope of the contact surface. Minor slopes indicate higher risk areas because of the greater surface of coastline exposed to hydrocarbon contamination. A beach, for example, offers a larger contamination area than a vertical cliff face.

- Wave movement. The other risk exposure factor that has been considered for coastline environments is wave movement, which has been subdivided into three classes: low, medium and high, with proportionally higher risk factors.

Impact values have been classified in relation to the possibility and difficulty of oil removal from the different types of substrata (clean up); the result depends mainly from the gravel size and/or roughness of the substrata. The greatest impact value has therefore been assigned to beach areas (sand/mud), where the only possible clean up option is usually to remove contaminated sand.

The qualitative aspects of environments have been examined in the damage indices, which serve to quantify the partial or total loss of natural, biological, anthropic or anthropogenic value.

In particular, the evaluation of damage indices for coastlines has taken into account natural and biological values in terms of water quality levels (low/high water quality), their degree of biodiversity (biodiversity value), productivity (biological productivity), and human presence, considering economic (presence of economic activities), aesthetic and recreational factors (recreational/landscape values) in the areas in question, for an overall result of ten categories.

\subsection{ESI for water stretches}

The second matrix has been drawn up for the evaluation of ESI for open waters, coastal waters, the seabed close to shore, marshes and wetlands. 
Two parameters have been considered in the risk exposure factor evaluation:

- Depth. The same number of risk classes have been assigned to the four pinpointed categories. Because physiochemical nature of the hydrocarbons, non water-soluble and with a lower density than seawater, the highest values goes with lowest depth.

- Wave movement. The same classification has been used as in Matrix 1, subdividing movement in low, average and high.

The physical sensitivity index has been calculated by adding the risk exposure factors to the impact values, subdivided according to whether clean up is necessary only on the sea surface or on the seabed as well; is impossible realize, with the actual techniques, a clean-up along the water column.

Damage indices have been pondered on a qualitative scale both according to their anthropic use and the naturalistic and biological value indices of each aquatic environment. The matrix therefore presents 10 damage categories, ranging from areas with low water quality to areas with high biodiversity values, considering also recreational value and economic issues such as shipping value, fishing/fish farming and aquaculture facilities.

\subsection{ESI for artificial features}

This part of the project is dedicated to the analysis of artificial elements on their possible accidental contact with hydrocarbons. The sensitivity that has been evaluated in these cases concerns the localization and complexity of those structures that could be involved in an accidental oil spill scenario. Both coastal and underwater structures have been taken into account.

The risk exposure factors were pondered according to three factors:

- Level referred to average sea level. Six intervals were pinpointed, with assigned numbers from one to four according to increasing contact frequency; the value 1 expresses situations where exposure to an accidental oil spill is least, such as the seabed over 10 metres deep. Values increase with lowest depth, where the contact probability with hydrocarbons increases;

- Contact. The second factor of risk evaluation is the position of the structural surface with respect to the oil exposure; for submerged buildings this would occur both horizontally and vertically, while for buildings above the water this could take place even mainly on vertical surfaces.

The impact value depends on the difficulty (medium/high) of removing polluting agents; these surfaces have an oil absorption capacity that is directly proportional to their conservation/age and roughness.

As far as anthropogenic/historical characteristics are concerned, the maximum damage index has been attributed to the loss of features with historic or architectural importance. The remaining classes, each subdivided into two categories (low and high), describe the various uses of the structures in question: presence of economic activities, presence of cultural functions or presence of service functions. 


\section{Results}

Not least among the results of the matrix analysis method, is the parameter clustering that includes the environmental, historical and cultural sensitivity of the milieu in question, and consequently the different environmental sensitivity indices

The values of impact and risk for physical sensitivity, both for natural or artificial features, have been calculated by summing the values of Risk Exposure factors with the Impact values. The matrix calculation of these results and the values of the damage indices provide the final integrated values (ESI).

For example, beaches with local bird life have an ESI value of 3, while those with recreational value have an ESI value of 2.

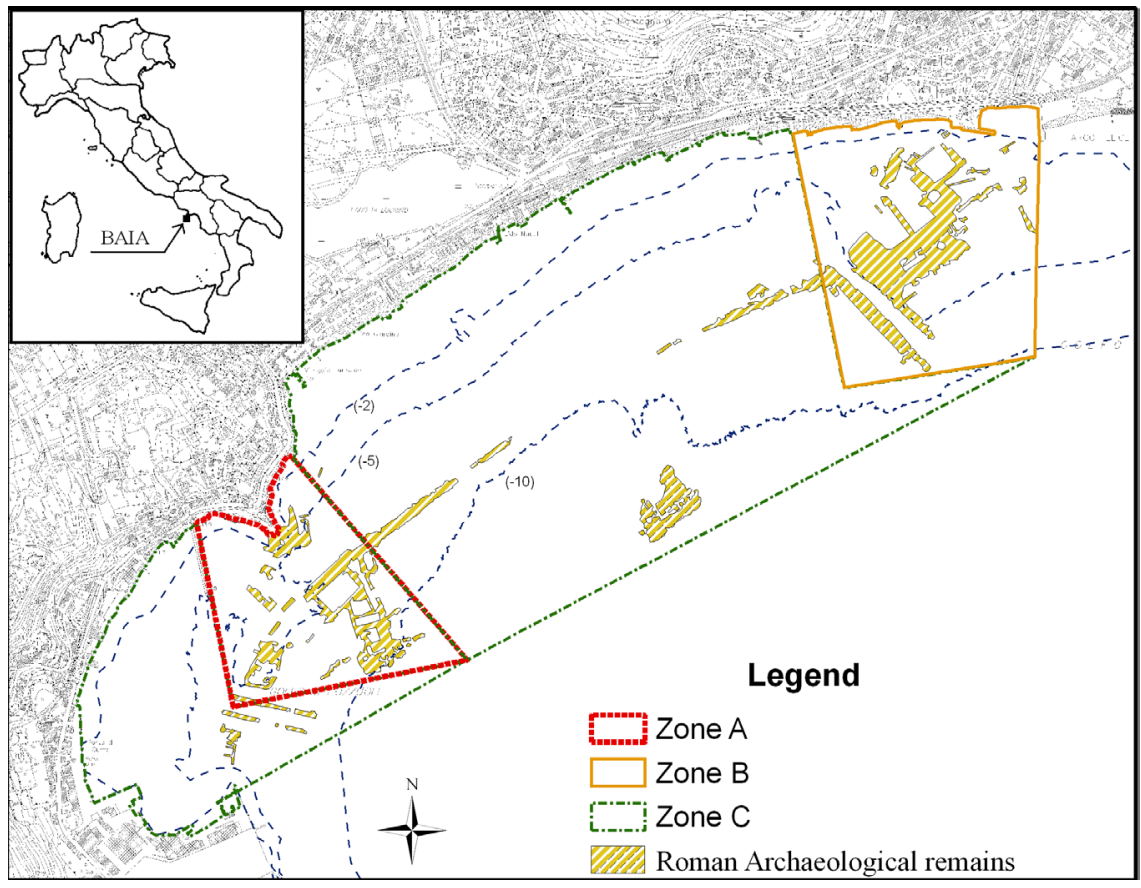

Figure 1: Underwater archaeological remains in the Submerged Natural and Archaeological Park of Baia.

\section{Discussions and conclusions}

In order to validate this method, a field test has been conducted choosing an area with a major complexity of different characteristics and difficulties. The case study contains all the elements corresponding to a complete use of the three matrices described above. The choice of the Submerged Natural and Archaeological Park of Baia in the gulf of Pozzuoli, fulfils the range of 
possibilities in the event of an extension of the mapping and data management method to the entire national coastline system.

Classified as a protected marine area by the Italian Ministry for the Environment, Land and Sea Protection in 2002 [5], Baia in fact contains copious archaeological remains, both above and below sea level, dating from the Roman Empire, when the area was part of the Campi Flegrei coastal settlements (Figure 1) and one of the smartest haunts of the imperial court. Over the centuries the Bradisismus effect concerning all of this volcanic area has provoked a sinking of the coastline and all the Roman remains (columns, statues, mosaics and streets) to about 6 meters below the water surface.

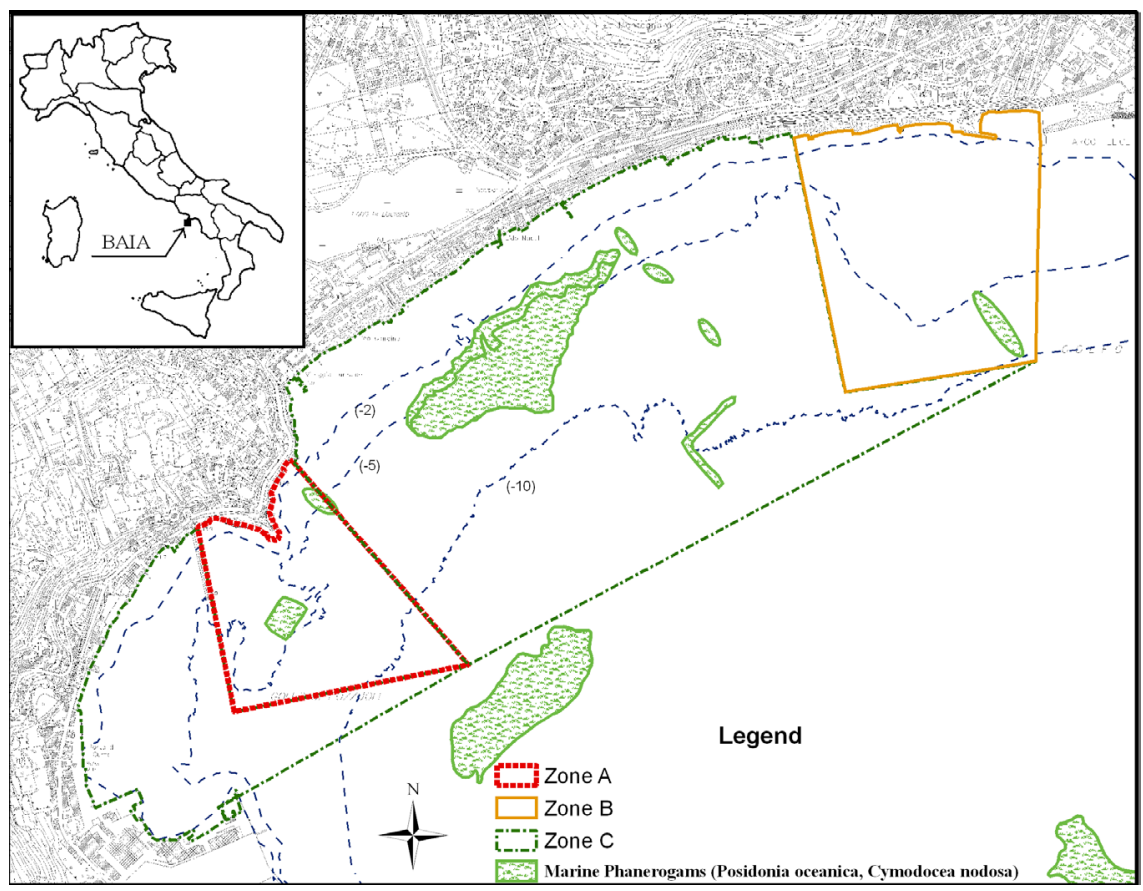

Figure 2: Marine phanerogams in the Submerged Natural and Archaeological Park of Baia.

The area is also a typical example of a Mediterranean marine ecosystem [6] (Figure 2), with a conspicuous presence of marine phanerogams grasslands and other relevant species and priority habitats listed both on the Barcelona Convention protocols and on the Natura 2000 EU directive.

Particularly during the summer months, the area is also exposed to a heavy tourist presence [7].

The ESI obtained from the analysis of the different scenarios of the case study will be used to create ESI maps highlighting areas with high vulnerability to possible oil spills. The long-term strategy is to apply the methodology to the entire Italian coastline, considering as "blanks" the coastal protected areas. 
The methodology will provide a clear system for localising environmental, landscape and cultural priorities for the protection of those areas that will results most vulnerable. This Geographic Information System is based on a complex database that includes all possible information that could be used in many different ways from local stakeholders, for different integrated management purposes.

\section{References}

[1] National Oceanic \& Atmospheric Administration (NOAA), National Ocean Service, Office of Response and Restoration. Sensitivity of Coastal Environments and Wildlife to Spilled Oil. Hawaii Atlas vol. I-II. 2001.

[2] National Oceanic \& Atmospheric Administration (NOAA) web site: http://response.restoration.noaa.gov/.

[3] CNR/IUAV. Venice Lagoon System Project. Final CD-rom of the research project. Venice, CIRCE, DAEST, STRATEMA, 1999.

[4] Cinquepalmi F., Schiuma D., Tagliapietra D., Benedetti C. and Zitelli A., Prevention of hydrocarbons sea pollution: Sensitivity Index Maps for the Venice Lagoon as integral component of oil-spill contingency planning and response. Environmental Coastal Regions. WIT Transaction of Ecology and the Environmental volume 25. WIT Press, 1998.

[5] Ministero dell'Ambiente e della Tutela del Territorio e del Mare. Parchi d'Italia. Il Sistema delle aree protette. Carsa Edizioni.

[6] CoNISMa (Consorzio Nazionale Interuniversitario per le Scienze del Mare) and SBAnace (Sopraintendenza per i Beni Archeologici di Napoli e Caserta). Caratterizzazione bio-ecologica e bionomica. Parco sommerso di Baia.

[7] Società Nazionale di Scienze, Lettere e Arti in Napoli. Ambiente marino costiero e territorio delle isole Flegree. Liguori Editore. 